\title{
Balantidium Coli: Rare Urinary Pathogen or Fecal Contaminant in Urine? Case Study And Review
}

\author{
Dr. Smita Gupta ${ }^{1}$,Dr. Purnima Bharati ${ }^{2}$,Dr. Kamlesh Prasad Sinha ${ }^{3}$, \\ Dr.R.K. Shrivastav ${ }^{4}$ \\ ${ }^{I}$ Tutor, Department Of Pathology, Rajendra Institute Of Medical Sciences, Ranchi, \\ Jharkhand, India \\ ${ }^{2}$ Junior Resident, Department Of Pathology, Rajendra Institute Of Medical Sciences, Ranchi, \\ Jharkhand, India \\ ${ }^{3}$ Associate Professor, Department Of Pathology, Rajendra Institute Of Medical Sciences, Ranchi, \\ Jharkhand, India \\ ${ }^{4}$ Professor, Department Of Pathology, Rajendra Institute Of Medical Sciences, Ranchi, \\ Jharkhand, India
}

\begin{abstract}
Balantidium coli is a known fecal pathogen causing dysentery, abdominal pain and weight loss. It can though rarely cause urinary tract infection. Differentiating between the two is very essential as many urinary samples received are contaminated with fecal pathogens. B. coli in urine should always alert the pathologist because urinary balantidiasis is in itself very rare, so fecal contamination must first be ruled out. We have seen two cases in 2 years where one of the case was suffering from urinary balantidiasis while the other was due to fecal contamination.
\end{abstract}

Keywords: Urinary balantidiasis, fecal contaminant, urine microscopy

\section{Introduction}

Balantidium coli is a parasitic species of ciliate alveolates that causes the disease balantidiasis ${ }^{1,2}$. It is the only member of the ciliate phylum known to be pathogenic to humans ${ }^{2,3}$. Balantidiasis is a zoonotic disease occurring in humans via the feco-oral route from the normal host, the pig. Water is the vehicle for most cases. Human-to-human transmission may also occur. Balantidium's habitats in humans are the cecum and colon where it causes mostly asymptomatic infections, or it may develop dysentery which is similar to that which is caused by Entamoeba histolytica. Rarely, the organism invades extraintestinal sites such as liver, lung, and genitourinary tract and causes infection ${ }^{3}$.

Case report 1-A 35-year-old male presented to Medicine Department of Rajendra Institute of Medical Sciences, Ranchi, with fever, malaise, anorexia of 28 days duration. A complete blood count and routine urine was requested and the samples were sent to our pathology department for assessment. The urine physically appeared smoky and turbid. Reaction was acidic and sugar and albumen were absent. Microscopic examination of the centrifuged sediment showed a 5-6 pus cells per HPF. Also, many ovoid to oblong ciliated parasites measuring approximately $70 \times 50 \mu \mathrm{m}$, were seen swimming rapidly across the slide The organism was covered with short, delicate uniform cilia (peritricate cilia) and several food vacuoles, macronuclei and a few ingested particles were present within cytoplasm. The morphology and swimming pattern were characteristic of B. coli(figure1) The complete hemogram of the patient showed mild neutrophilia. Serology done for HIV and HbsAg were negative. His kidney functions tests, LFT and blood sugar were found to be within normal limits. In order to rule out fecal contamination, a repeat MSU sample and morning fecal sample were taken.MSU samples showed similar organisms and fecal sample examined for ova cysts and parasites were found to be negative. The patient was confirmed too have UTI due to B.coli and metronnidazole was subscribed.

Case report 2-A 56-year-old female presented to Medicine Department of Rajendra Institute of Medical Sciences, Ranchi, with anorexia, weakness and on and off diarrhea of 2 years duration. Complete blood count and routine urine samples were sent to our pathology department for assessment. The urine was pale yellow and reaction was acidic. Sugar and albumen were absent. Microscopic examination of the centrifuged sediment showed morphology and swimming pattern characteristic of B. coli(figure 2). The complete hemogram of the patient showed mild microcytic hypochromic anemia with $\mathrm{Hb}$ value being $9.6 \mathrm{mg} / \mathrm{dl}$. Morning stool samples were also sent and similar findings along with ova cysts were found. Occult blood was also positive. His kidney functions tests, LFT and blood sugar were found to be within normal limits. Interestingly, repeat MSU urine sample was found to be negative for the organism, while stool sample continued to be positive for 2 days. Thus patient was negative for urinary Balantidiasis and Fecal contamination of urine was confirmed. 


\section{Figures}

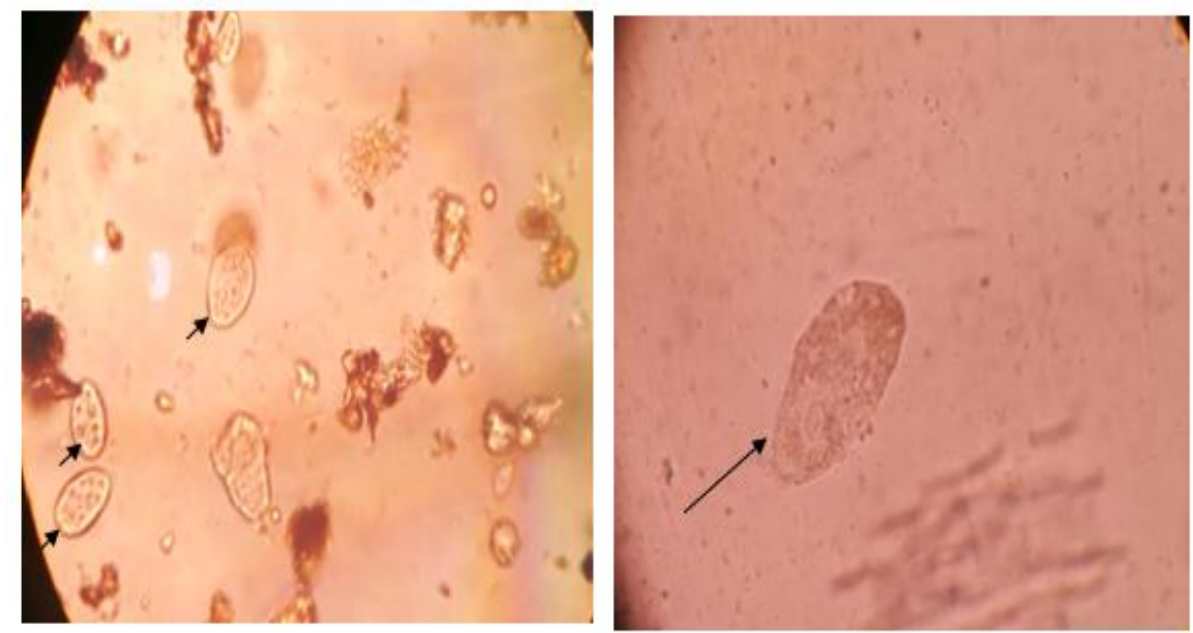

Fig 1.- (wet film microscopy of urine). Oval-to-oblong ciliated trophozoites(a-at low power,b-at higher power).Faint cilia and a nucleus can be seen in $1 b$ (arrow)
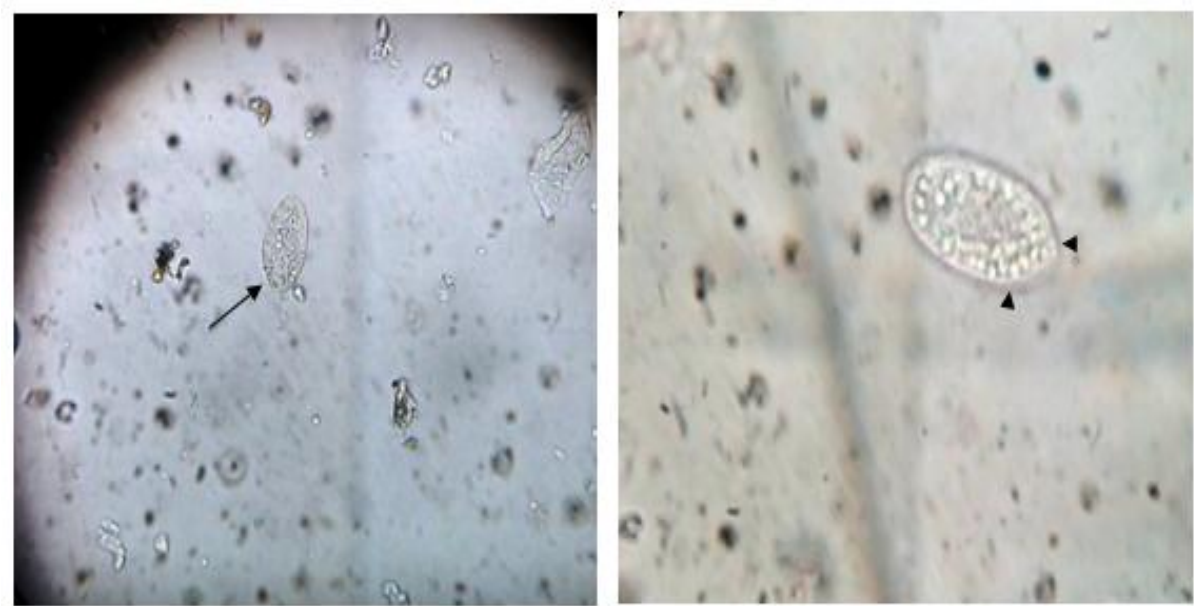

Fig.2-Similar findings in $2^{\text {nd }}$ patient in low power(a) and high power(b).Multiple food vacuoles can be easily seen at high power(arrow heads)

\section{Discussion}

Balantidiasis is a zoonotic disease which is caused by Balantidium coli (B. colī and it is acquired by humans via the fecal-oral route. It was first recognized by Malmsten in two humans with dysentery in the year $1857^{1,3}$. B. coli is the largest and only ciliated protozoan which is known to infect humans. Pigs are the reservoir hosts and they usually remain asymptomatic. Even after infection, very few patients show the classical symptoms and develop dysentery which is similar to that caused by Entamoeba histolytica. It is found worldwide but is mostly found in developing countries, where water sources may be contaminated with porcine or human faeces ${ }^{1}$. Genitourinary sites of infection, including uterine infection, vaginitis, and cystitis, are thought to occur via direct spread from the anal area or as secondary to rectovaginal fistulas which are created due to infection with B. coli ${ }^{1}$.

Balantidium has a simple life cycle. Cyst is the infective stage, when ingested liberates trophozoites in the large intestine, which may remain in the bowel or may invade submucosal coat of the bowel. They multiply by binary fission. The trophozoites and cysts of B. coli are shed in feces and if the cysts contaminate drinking water or food, the infection can be spread to other pigs or humans. No intermediate host is needed. Morphologically, the trophozoite measures 30-150 $\mu \mathrm{m}$ in length and $25-120 \mu \mathrm{m}$ in width; the cyst, which may be spherical or slightly ovoid, measures $40-60 \mu \mathrm{m}$ in diameter. The mouth (oral apparatus) is located at the tapering anterior end, and the cytopyge (anus) is located at the rounded posterior end. A groove leading to mouth is noted at the anterior end. It contains two nuclei and many cytoplasmic vacuoles. The body is covered with a delicate pellicle showing longitudinal striations. The cilia are short and delicate and are of uniform length. 
Making a lab diagnosis is relatively easy, because of its large size and spiral motility. Differentiation from Trichomonas vaginalis and Entamoeba histolytica is important, but relatively easy. Balantidial cysts are 40-60 microns in diameter and they are binucleate, in contrast to E. histolytica cysts which are smaller 10-20 microns and are quadrinucleate ${ }^{1}$. Trophozoite stage of E. histolytica moves on the slide surface by means of an anterior ectoplasmic pseudopod and is smaller (around $25 \mu \mathrm{m}$ ) in diameter. Trichomonas is flagellate, not ciliate organism, exists only as trphozoite stage, and roughly measures $9-12 \mu$. In this patient, excellent morphology of the parasite could be demonstrated in the urine sample by light microscopy. The only other ciliated parasite which has a similar morphology is Paramecium, which is non pathogenic and it can be found in contaminated water. It has rarely been reported to colonize urinary tracts of dialysis patients ${ }^{5}$. As our patient was symptomatic the organism was more likely to be B.coli in our case. Tetracycline and Metronidazole are drugs of choice for B.coli.

B.coli in our first case may have invaded the urinary bladder through the colonic mucosa to or directly through the anal area. Although in immuno compromised individuals, malnutrition and alcoholism are important risk factors for balantidiosis, our patient was HIV negative and there was no history of either malnutrition or alcoholism $^{6,7}$.

\section{Conclusion}

To conclude, B.coli is a rare urinary pathogen that can be found throughout the world. Microscopic examination of Mid stream urine sample can help in easily diagnosing this large parasite, based on its characteristic morphology and rapid spiralling motility. Before reporting a case of urinary balantidiosis, fecal contamination must always be ruled out because of rarity of such case. Clean water and hygienic sanitary conditions are the most efficient strategis to prevent human infections.

\section{References}

[1]. Schuster FL, Ramirez-Avila L. Current world status of Balantidium coli. Clin Microbiol Rev 2008;21:626-38.

[2]. Ramachandran, Ambili (23 May 2003). "Introduction". The Parasite: Balantidium coli The Disease: Balantidiasis. ParaSite. Stanford University.

[3]. Malmsten PH. Infusorien als Intestinal-Thiere beim Menschen. Arch Pathol Anat Physiol Klin Med 1857;12:302-9

[4]. Chatterjee KD. Parasitology. $13^{\text {th }}$ ed. New Delhi: CBS Publishers \& Distributors Pvt. Ltd.; 2009. p. 138-40

[5]. Singh S, Dash SC. Paramecium colonizing urinary tract of a patient on dialysis: a rare entity. Nephron.1992;62(2):243-4

[6]. Anargyrou K, Petrikkos GL, Suller MT, Skiada A, Siakantaris MP, Osuntoyinbo RT, et al. Pulmonary Balantidium coli infection in a leukemic patient. Am J Hematol. 2003;73(3):180-3.

[7]. Cermeno JR, Hernandez De Cuesta I, Uzcategui O, Paez J, Rivera M, Baliachi N. Balantidium coli in an HIV-infected patient with chronic diarrhoea. AIDS. 2003;17(6):941-2. 